\title{
REDUCTION NUMBERS AND REES ALGEBRAS OF POWERS OF AN IDEAL
}

\author{
LÊ TUÂN HOA
}

(Communicated by Louis J. Ratliff, Jr.)

\begin{abstract}
Let $I$ be an ideal in a Noetherian local ring $(R, \mathfrak{m})$. It is shown that for $n \gg 0$ the reduction number $r_{J}\left(I^{n}\right)$ of $I^{n}$ with respect to a minimal reduction $J$ is not only independent from the choice of $J$ but also is stable. If $I$ is an m-primary ideal, we give a criterion for the Rees algebra $R\left[I^{n} t\right]$ with $n \gg 0$ to be Cohen-Macaulay.
\end{abstract}

\section{INTRODUCTION}

Let $(R, \mathfrak{m})$ denote a local Noetherian ring with an infinite residue field $R / \mathfrak{m}$. If $I$ is an ideal in $R$, recall that an ideal $J \subseteq I$ is called a reduction of $I$ if $I^{n+1}=J I^{n}$ for some nonnegative integer $n$. A reduction $J$ is called a minimal reduction if it does not properly contain a reduction of $I$. These concepts were introduced and studied by Northcott and Rees [11]. If $J$ is a minimal reduction of $I$, we define the reduction number of $I$ with respect to $J$, denoted by $r_{J}(I)$, to be the least nonnegative integer $n$ such that $I^{n+1}=J I^{n}$. The reduction number of $I$ is defined by $r(I)=\min \left\{r_{J}(I) ; J \subseteq I\right.$ is a minimal reduction of I\}.

The reduction number was introduced by Sally [14] in order to study CohenMacaulayness of the associated graded ring $G(I)=\bigoplus_{n \geq 0} I^{n} / I^{n+1}$ of $R$ with respect to $I$. For the usefulness of this notion see $[3,4,6,8-10,15,17,19]$. In general it is hard to calculate $r(I)$. More recently, the question, due to Sally [15], when is $r_{J}(I)$ independent from $J$, attained much attention. Some partial solutions of this problem were given in $[8,9,17,19]$. The crucial point of Trung's approach in [19] is a relationship between the reduction number $r_{J}(I)$ and certain cohomologically defined invariants of $G(I)$-among them the Castelnuovo-Mumford regularity (see the inequalities $(*)$ ).

The main goal of this paper is to study the asymptotic property of $r_{J}\left(I^{n}\right)$. By combining Trung's approach and an idea of Herrmann, Ribbe, and Zarzuela (see [6, Proposition 2.6]) we get a surprising result (Theorem 2.1). This theorem states that for any ideal $I$ of $R$ the reduction number $r_{J}\left(I^{n}\right)$ is not only independent from $J$ but also stable if $n \gg 0$. Moreover, we can compute the

Received by the editors September 25, 1991 and, in revised form, March 2, 1992.

1991 Mathematics Subject Classification. Primary 13A15; Secondary 13A30, 13H10.

Key words and phrases. Minimal reduction, reduction number, associated graded ring, Rees algebra, Cohen-Macaulay ring. 
asymptotic value of $r_{J}\left(I^{n}\right)$. As a byproduct we give a criterion for the Rees algebra $R\left[I^{n} t\right]=R \oplus I^{n} t \oplus I^{2 n} t^{2} \oplus \cdots$ of $I^{n}$ to be a Cohen-Macaulay (abbr. C-M) ring for $n \gg 0$, where $I$ is an m-primary ideal (Theorem 3.1). Of course, we can apply a general Cohen-Macaulayness criterion of [10] to $R\left[I^{n} t\right]$; however, we must then calculate local cohomology modules of $G\left(I^{n}\right)$ for each $n$. Our result is free from that and therefore is of interest.

The paper is divided into three sections. In $\S 2$ we give the proof of the main Theorem 2.1 and its consequences. Section 3 is devoted to Cohen-Macaulayness of $R\left[I^{n} t\right]$.

Finally I would like to thank the referee for some suggestions.

\section{REDUCTION NUMBERS}

A Noetherian graded ring $A=\bigoplus_{n>0} A_{n}$ is called a (standard) graded $R$ algebra if $A_{0}=R$ and $A$ is generated by $A_{1}$ over $R$. In this paper $R$ will also be regarded as a graded $R$-algebra concentrated in degree 0 . For a graded $A$-module $M=\bigoplus_{n \in Z} M_{n}$ we denote by $[M]_{n}$ the $n$th graded piece of $M$, i.e., $[M]_{n}=M_{n}$. $M(p)$ denotes the same module $M$ shifted by $p$, i.e., $[M(p)]_{n}=M_{n+p}$. The local cohomology module $H_{A_{+}}^{i}(M), i \in Z$, of $M$ with support in $A_{+}=\bigoplus_{n>0} A_{n}$ are also graded $A$-modules. Concerning local cohomology theory of graded modules see [5]. We set

$$
a_{i}(M)=\sup \left\{n \in Z ;\left[H_{A_{+}}^{i}(M)\right]_{n} \neq 0\right\}
$$

and

$$
e_{i}(M)=\inf \left\{n \in Z ;\left[H_{A_{+}}^{i}(M)\right]_{n} \neq 0\right\} .
$$

(Convention: if $H_{A_{+}}^{i}(M)=0$ we set $a_{i}(M)=-\infty$ and $e_{i}(M)=+\infty$.) If $R=K$ is a field and $\operatorname{dim} M=d$ then $a_{d}(M)$ is just the so-called $a$-invariant of $M$ introduced in [5] and plays an important role in the study of Gorenstein rings (see, e.g., $[5,6])$. The number

$$
\operatorname{reg} M=\max \left\{a_{i}(M)+i ; i \in Z\right\}
$$

is called the Castelnuovo-Mumford regularity of $M$ (see [2, 12]).

Now let $I \subseteq \mathfrak{m}$ be an arbitrary ideal of $R$. We shall also denote by $G$ and $G^{\prime}$ the associated graded rings $G(I)$ and $G\left(I^{n}\right)$, respectively. Set $s=s(I)=$ $\operatorname{dim} G(I) \otimes R / \mathfrak{m}$ the analytic spread of $I$ [11]. It is well known that $s(I)$ is equal to the minimum number of generators of every minimal reduction of $I$. Let $J$ be a minimal reduction of $I$. Then we get from [19, Proposition 3.2] the following relationship between $r_{J}(I)$ and $a_{i}(G(I))$ :

$$
a_{s}(G(I))+s \leq r_{J}(I) \leq \operatorname{reg} G(I) .
$$

Our aim in this section is to prove the following main result.

Theorem 2.1. Let $I \subseteq \mathfrak{m}$ be an arbitrary ideal of $R$. Then $r_{J}\left(I^{n}\right)$ is independent of $J$ and stable if $n \gg 0$. Namely, for all $n>\max \left\{\left|a_{i}(G(I))\right| ; a_{i}(G(I)) \neq-\infty\right\}$ we have

$$
r_{J}\left(I^{n}\right)= \begin{cases}s & \text { if } a_{s}(G(I)) \geq 0 \\ s-1 & \text { if } a_{s}(G(I))<0\end{cases}
$$

where $J$ is any minimal reduction of $I^{n}$.

We shall need some auxiliary results. Let $A$ be a graded $R$-algebra and $M$ be a graded $A$-module. The following result was essentially proven in [12, Theorem 2]. 
Lemma 2.2. Assume that $d=\sup \left\{i ; H_{A_{+}}^{i}(M) \neq 0\right\}>0$ and $\left[H_{A_{+}}^{d}(M)\right]_{n}=0$ for an integer $n$. Then $\left[H_{A_{+}}^{d}(M)\right]_{m}=0$ for all $m \geq n$.

The following result is perhaps known. We prove it for the sake of completeness.

Lemma 2.3. $s(I)=\sup \left\{i ; H_{G(I)_{+}}^{i}(G(I)) \neq 0\right\}$.

Proof. By induction on $s:=s(I)$ one can easily show that the number on the right side is less than or equal to $s$. One has still to show that $H_{G_{+}}^{s}(G) \neq 0$. From the exact sequence of $G$-modules

$$
0 \rightarrow \mathfrak{m} G \rightarrow G \rightarrow G / \mathfrak{m} G \rightarrow 0,
$$

we get an epimorphism

$$
H_{G_{+}}^{s}(G) \rightarrow H_{G_{+}}^{s}(G / \mathfrak{m} G) \rightarrow 0 .
$$

Since $s=\operatorname{dim}(G / \mathfrak{m} G)$ and $(G / \mathfrak{m} G)_{+}$is the maximal homogeneous ideal of $G / \mathfrak{m} G$, we have $H_{G_{+}}^{s}(G / \mathfrak{m} G) \cong H_{(G / \mathrm{m} G)_{+}}^{s}(G / \mathfrak{m} G) \neq 0$. Hence $H_{G_{+}}^{s}(G) \neq 0$.

Note that $s\left(I^{n}\right)=s(I)$. The following lemma is a key result.

Lemma 2.4. Assume that $s=s(I) \geq 1$. Then we have, for all $i \leq s$ and $n \geq 1$,

(i) $a_{i}\left(G\left(I^{n}\right)\right) \leq\left[a_{i}(G(I)) / n\right]$,

(ii) $e_{i}\left(G\left(I^{n}\right)\right) \geq\left[e_{i}(G(I)) / n\right]$, and

(iii) $a_{s}\left(G\left(I^{n}\right)\right)=\left[a_{s}(G(I)) / n\right]$,

where $[a]=\max \{m \in Z ; m \leq a\}$.

Remark. This lemma is related to a result of Herrmann et al. Let $I$ be an m-primary ideal, i.e., $s=d$. Then $a_{d}(G)$ and $a_{d}\left(G^{\prime}\right)$ are the $a$-invariants of $G$ and $G^{\prime}$, respectively. In this case the equality (iii) was established in [6, Proposition 2.6] under the assumption that $G$ is C-M. By using Lemma 2.2 we do not need here the C-M assumption on $G$.

Recall that the Veronesian embedding of a graded $R$-algebra $A$ is defined as $A^{(k)}=\bigoplus_{n \geq 0} A_{n k} \quad(k$ is a positive integer). If $M$ is a graded $A$-module, then the Veronesian embedding $M^{(k)}=\bigoplus_{n \in Z} M_{n k}$ of $M$ is a graded $A^{(k)}$-module.

Proof of Lemma 2.4. The proof of (i) and (ii) is essentially the same as the ones of [6, Proposition 2.6]. It follows from a cohomological exact sequence derived from the following short exact sequence of $R\left[I^{n} t\right]$-modules

$$
0 \rightarrow I^{n-t+1} G^{\prime} \rightarrow I^{n-t} G^{\prime} \rightarrow(G(n-t))^{(n)} \rightarrow 0,
$$

where $t=1, \ldots, n$ (we take here the local cohomology with support in $R\left[I^{n} t\right]_{+}$). Note that for the first part the argument in the proof of [6, Proposition 2.6] did not really use that $G$ is C-M.

To finish the proof one needs still to show that $a_{s}\left(G^{\prime}\right) \geq\left[a_{s} / n\right]$, where $a_{s}=a_{s}(G)$. To this end consider the above exact sequence with $t=n$. Note that $I G^{\prime}$ can be also considered as a $G^{\prime}$-module. We have

$$
H_{R\left[I^{n}\right]_{+}}^{s}\left(G^{\prime}\right) \cong H_{G_{+}^{\prime}}^{s}\left(G^{\prime}\right) \text { and } H_{R\left[I^{n}\right]_{+}}^{s+1}\left(I G^{\prime}\right) \cong H_{G_{+}^{\prime}}^{s+1}\left(I G^{\prime}\right)=0 .
$$

By [5, Theorem 3.1.1]

$$
H_{R\left[I^{n}\right]_{+}}^{s}\left(G^{(n)}\right) \cong\left(H_{R\left[I^{n} t\right]_{+}}^{s}(G)\right)^{(n)} \cong\left(H_{G_{+}^{\prime}}^{s}(G)\right)^{(n)} .
$$


Hence we get an epimorphism

$$
\left[H_{G_{+}^{\prime}}^{s}\left(G^{\prime}\right)\right]_{j} \rightarrow\left[H_{G_{+}}^{s}(G)\right]_{n j} \rightarrow 0 .
$$

Let $j=\left[a_{s} / n\right]$. Then $n j \leq a_{s}$. Since $\left[H_{G_{+}}^{s}(G)\right]_{a_{s}} \neq 0$ (by the definition of $a_{s}$ ), we get from Lemma 2.2 that $\left[H_{G_{+}}^{s}(G)\right]_{n j} \neq 0$. Hence $\left[H_{G_{+}^{\prime}}^{s}\left(G^{\prime}\right)\right]_{j} \neq 0$, which gives $a_{s}\left(G^{\prime}\right) \geq\left[a_{s} / n\right]$, as required.

Proof of Theorem 2.1. The case $s=0$ is trivial. Let $s \geq 1$ and $n>\max \left\{\left|a_{i}(G)\right|\right.$; $\left.a_{i}(G) \neq-\infty\right\}$. By Lemma 2.3, $a_{s}(G) \neq-\infty$. From Lemma 2.4(i) and (iii) we then get $a_{i}\left(G^{\prime}\right) \leq 0$ for $i=0, \ldots, s-1$ and

$$
a_{s}\left(G^{\prime}\right)= \begin{cases}0 & \text { if } a_{s}(G) \geq 0 \\ -1 & \text { if } a_{s}(G)<0 .\end{cases}
$$

Hence

$$
\operatorname{reg} G^{\prime}=a_{s}\left(G^{\prime}\right)+s= \begin{cases}s & \text { if } a_{s}(G) \geq 0 \\ s-1 & \text { if } a_{s}(G)<0\end{cases}
$$

Now the theorem follows from $(*)$.

Example 2.5. Let $(R, \mathfrak{m})$ be a $d$-dimensional generalized $C$ - $M$ ring, i.e., a ring with $l\left(H_{\mathfrak{m}}^{i}(R)\right)<\infty$ for all $i<d$ (see, e.g., [18]). Let $x_{1}, \ldots, x_{d}$ be a standard system of parameters of $R$, i.e., a system of parameters satisfying $\left(x_{1}, \ldots, x_{d}\right) H_{\mathrm{m}}^{i}\left(R /\left(x_{1}, \ldots, x_{j}\right) R\right)=0$ for all $i, j$ with $i+j<d$ (see, e.g., $[1,18])$. Set $I=\left(x_{1}, \ldots, x_{d}\right) R$. Then $a_{d}(G)=-d$ or $-(d+1)$ and for any minimal reduction $J$ of $I^{n}(n \geq 2)$ we have $r_{J}\left(I^{n}\right)=\left[a_{d}(G) / n\right]+d$. If, in addition, $R$ is C-M then $a_{d}(G)=-d$. In particular, if $R$ is a regular ring then $r\left(\mathfrak{m}^{n}\right) \leq 1$ if and only if $n=1$ or $d=2$ or $(d, n)=(3,2)$ (cf. [13, Example 2.3]).

Proof. We have $s(I)=d$. It is known that $H_{G_{+}}^{i}(G) \cong H_{\mathfrak{m}}^{i}(R)(i)$ for $i=$ $0, \ldots, d-1$ and $\left[H_{G_{+}}^{d}(G)\right]_{j}=0$ for $j>-d$ (see [18, Theorem 5.4]). That means $a_{i}(G)=-i$ or $-\infty$ for $i=0, \ldots, d-1$ and $a_{d}(G) \leq-d$. We will show that $a_{d}(G) \geq-d-1$. Let $x_{1}^{*}$ be the initial form of $x_{1}$ in $G$. As in the proof of [18, Theorem 5.4] we may assume that $\operatorname{depth} R>0$. Then $x_{1}^{*}$ is a non-zero-divisor of $G$. Using the exact sequence

$$
0 \rightarrow G(-1) \stackrel{x_{1}^{*}}{\longrightarrow} G \rightarrow G / x_{1}^{*} G \cong G\left(\left(x_{2}, \ldots, x_{d}\right)\left(R / x_{1} R\right)\right) \rightarrow 0
$$

and Lemma 2.2 we can show by induction that $a_{d}(G) \geq-d-1$ (resp. $a_{d}(G) \geq$ $-d$ in the C-M case). Hence $a_{d}(G)=-d$ or $-d-1$. Now applying Lemma 2.4 and $(*)$ we get $r_{J}\left(I^{n}\right)=\left[a_{d}(G) / n\right]+d$ for $n \geq 2$.

An ideal $I$ with $r(I) \leq 1$ deserves special interest. It is related to various notions of stable ideals (see, e.g., $[13,16])$. In some sense the following immediate consequence of Theorem 2.1 generalizes [16, Theorem 2.9].

Corollary 2.6. Assume that $s(I) \leq 1$. Then $r\left(I^{n}\right) \leq 1$ for $n \gg 0$.

This corollary can also be obtained from the following lemma.

Lemma 2.7. $\left.r\left(I^{n}\right) \leq\right] \frac{r(I)+1-s(I)}{n}[+s(I)-1$, where $] a[=\min \{m \in Z ; m \geq a\}$. Proof. Set $r=r\left(I^{n}\right), s=s(I)$, and $\left.p=\right](r+1-s) / n[+s-1$. The case $s=0$ is trivial (in this case we get even the equality). Let $s \geq 1$. Then 
$q:=n(p+1)-(s(n-1)+1) \geq r$. Let $x_{1}, \ldots, x_{s}$ be a minimal basis of a minimal reduction of $I$ such that $I^{r+1}=\left(x_{1}, \ldots, x_{s}\right) I^{r}$.

Claim. For all $s \geq 1$ and $k \geq 0$ we have that

$$
\left(x_{1}, \ldots, x_{s}\right)^{s k+1} \subseteq\left(x_{1}^{k+1}, \ldots, x_{s}^{k+1}\right)\left(x_{1}, \ldots, x_{s}\right)^{s k-k}
$$

Proof. We have

$$
\left(x_{1}, \ldots, x_{s}\right)^{s k+1}=\left(x_{1}^{s k+1}, x_{1}^{s k} x_{2}, \ldots, x_{s}^{s k+1}\right) .
$$

Since the total degree of each monomial on the right side is $s k+1$, at least one $x_{i}$ has the degree $\geq k+1$. Hence

$$
\left(x_{1}, \ldots, x_{s}\right)^{s k+1} \subseteq\left(x_{1}^{k+1}, \ldots, x_{s}^{k+1}\right)\left(x_{1}, \ldots, x_{s}\right)^{s k-k}
$$

as required.

Now we have

$$
\begin{aligned}
\left(I^{n}\right)^{p+1} & =I^{n(p+1)}=\left(x_{1}, \ldots, x_{s}\right)^{s(n-1)+1} I^{q} \\
& \subseteq\left(x_{1}^{n}, \ldots, x_{s}^{n}\right)\left(x_{1}, \ldots, x_{s}\right)^{s(n-1)-n+1} I^{q} \quad \text { [by the claim] } \\
& \subseteq\left(x_{1}^{n}, \ldots, x_{s}^{n}\right)\left(I^{n}\right)^{p} \subseteq\left(I^{n}\right)^{p+1} .
\end{aligned}
$$

Hence $r\left(I^{n}\right) \leq p$.

As another consequence of this lemma we get: let $I$ be an ideal with $s(I) \leq$ 2 . If $r(I) \leq 1$ then $r\left(I^{n}\right) \leq 1$ for all $n>0$. Since $s(I) \leq \operatorname{dim} R$, this gives an extension of [13, Corollary 4.3].

\section{ReEs Algebras}

In this section let $(R, \mathfrak{m})$ be a $d$-dimensional local ring and $I$ an $\mathfrak{m}$-primary ideal.

Theorem 3.1. (i) Assume that $G(I)$ is a generalized $C-M$ ring with $a_{i}(G(I))<0$ for all $i=0, \ldots, d$. Then $R\left[I^{n} t\right]$ is $C-M$ for all $n \gg 0$.

(ii) If $R\left[I^{n} t\right]$ is $C-M$ for some $n>0$ then $G(I)$ is a generalized $C-M$ ring with $a_{d}(G(I))<0$.

Proof. Note that $s(I)=d=\operatorname{dim} G$ and $H_{G_{+}}^{i}(G) \cong H_{P}^{i}(G)$, where $P=\mathfrak{m} / I \oplus G_{+}$ denotes the maximal homogeneous ideal of $G$.

(i) Since $G$ is generalized C-M, we have $\left[H_{G_{+}}^{i}(G)\right]_{j}=0$ for $j \ll 0$ and $i=0, \ldots, d-1$, i.e., $e_{0}(G)>-\infty, \ldots, e_{d-1}(G)>-\infty$. Let

$$
n_{0}=\max \left\{-a_{d}(G),-e_{0}(G), \ldots,-e_{d-1}(G)\right\} .
$$

From the assumption $a_{i}(G)<0$ and from Lemma 2.4 we get for all $n>n_{0}$ that

$$
\left[H_{G_{+}^{\prime}}^{i}\left(G^{\prime}\right)\right]_{j}=0 \text { for } j \neq-1, i=0, \ldots, d-1
$$

and

$$
\left[H_{G_{+}^{\prime}}^{d}\left(G^{\prime}\right)\right]_{j}=0 \text { for } j \geq 0 .
$$

By [10, Theorem 1.1] $R\left[I^{n} t\right]$ is a C-M ring.

(ii) Assume that $R\left[I^{n} t\right]$ is C-M. Since $R\left[I^{n} t\right] \cong(R[I t])^{(n)}$, it follows from [1, Lemma 6.5] that $R[I t]$ is generalized C-M. Hence, by [10, Proposition 3.3], 
we get that $G$ is a generalized C-M ring. Further, in the proof of Lemma 2.4 we have the following epimorphism:

$$
\left[H_{G_{+}^{\prime}}^{d}\left(G^{\prime}\right)\right]_{j} \rightarrow\left[H_{G_{+}}^{d}(G)\right]_{n j} \rightarrow 0 .
$$

Let $j=0$. Since $R\left[I^{n} t\right]$ is C-M, again by [10, Theorem 1.1], $\left[H_{G_{+}^{\prime}}^{d}\left(G^{\prime}\right)\right]_{0}=0$. Hence $\left[H_{G_{+}}^{d}(G)\right]_{0}=0$. By Lemma 2.2 we must have $a_{d}(G)<0$.

Corollary 3.2. Assume that $G(I)$ is $C-M$. Then the following conditions are equivalent:

(i) $R\left[I^{n} t\right]$ is a C-M ring for some $n>0$.

(ii) $R\left[I^{n} t\right]$ is a $C-M$ ring for all $n>0$.

(iii) $a_{d}(G(I))<0$.

Proof. (ii) $\Rightarrow$ (i) is trivial. (i) $\Rightarrow$ (iii) follows from Theorem 3.1. Assume (iii). Then by $\left[10\right.$, Theorem 1.1] $R[I t]$ is C-M. Hence $R\left[I^{n} t\right] \cong(R[I t])^{(n)}$ is C-M for all $n>0$.

Example 3.3 (cf. [7, Proposition 3.1]). Let $K$ be an infinite field and $A=$ $K\left[x_{1}, \ldots, x_{n}\right]$ be a graded $d$-dimensional $K$-algebra. Let

$$
F: 0 \rightarrow \bigoplus_{i} S\left(-d_{g i}\right) \rightarrow \cdots \rightarrow \bigoplus_{i} S\left(-d_{1 i}\right) \rightarrow S \rightarrow A \rightarrow 0
$$

be a minimal graded free resolution of $A$, where $S=K\left[X_{1}, \ldots, X_{n}\right]$. Suppose that $A$ is C-M. Let $(R, \mathfrak{m})$ denote the $A_{+}$-adic completion of $A$. We set $d_{g}=\max _{i}\left\{d_{g i}\right\}$. Then $R\left[\mathfrak{m}^{q} t\right]$ is C-M for some $q>0$ (or for all $q>0$ ) if and only if $d_{g} \leq n-1$. This gives the correct form for [7, Proposition 3.1] and was in fact proven there (there was a little confusion in its proof: the reduction number of $\mathfrak{m}$ defined in [7] is not equal but one greater than the maximal degree of the homogeneous socle elements of $A /\left(a_{1}, \ldots, a_{d}\right) A$, where $a_{1}, \ldots, a_{d}$ is a system of parameters of 1 -forms).

Indeed, in this case $G(\mathfrak{m}) \cong A$ is a C-M ring. Hence, by Corollary 3.2 , one has only to show that $a_{d}(G)<0$ if and only if $d_{g} \leq n-1$. But this is immediate from the relation $n=d+g$ and from the following result of Eisenbud and Goto [2]: $d_{g}-g=\operatorname{reg} A=\operatorname{reg} G=a_{d}+d$ (since $G$ is C-M).

Next we give a sharpening of a result of Goto and Shimoda [4].

Proposition 3.4. If $R\left[\mathrm{~m}^{n} t\right]$ is $C-M$ then $R$ satisfies Serre's condition $\left(S_{2}\right)$, i.e., depth $R_{\mathfrak{p}} \geq \min (2, \mathrm{ht} \mathfrak{p})$ for all $\mathfrak{p} \in \operatorname{Spec} R$.

Proof. By [10, Lemma 4.1] $R$ is a generalized C-M ring. Hence $R$ is a locally C-M ring, i.e., $R_{\mathfrak{p}}$ is C-M for all $\mathfrak{p} \neq \mathfrak{m}$; therefore, it suffices to show that depth $R \geq 2$. Since $R\left[\mathfrak{m}^{n} t\right]$ is C-M, it is known that depth $R>0$ (see, e.g., [10, Corollary 3.4]). Let $\left(a_{1}, \ldots, a_{d}\right) R$ be a minimal reduction of $\mathfrak{m} . a_{1}, \ldots, a_{d}$ forms, of course, a system of parameters of $R$. Then $a_{1}$ is a nonzero divisor of $R$ (see, e.g., [3, Lemma 2.4]). Assume that $a_{2} x=a_{1} y$ for some $x, y \in \mathrm{m}$. Analyzing the proof of Lemma 2.7 we get that $\left(a_{1}^{n}, \ldots, a_{d}^{n}\right) R$ is a minimal reduction of $\mathfrak{m}^{n}$. Hence the sequence $a_{1}, a_{2}-a_{1}^{n} t, \ldots, a_{d}-a_{d-1}^{n} t, a_{d}^{n} t$ is a system of parameters of $R\left[\mathfrak{m}^{n} t\right]$. Since $x, y \in \mathfrak{m}$, we have

$$
x\left(a_{2}-a_{1}^{n} t\right)=a_{1}\left(y-a_{1}^{n-1} x t\right) \in a_{1} R\left[\mathfrak{m}^{n} t\right] .
$$


From Cohen-Macaulayness of $R\left[\mathfrak{m}^{n} t\right]$ it then follows that $x \in a_{1} R$. This means $a_{1}, a_{2}$ is a regular sequence of $R$, as required.

Finally we will derive some consequences for the case of 2-dimensional local rings.

Corollary 3.5. Assume that $(R, \mathfrak{m})$ is a 2-dimensional $C-M$ ring. Then $R\left[I^{n} t\right]$ is $C-M$ for $n \gg 0$ if and only if $a_{2}(G(I))<0$.

Proof. The necessary part follows from Theorem 3.1. Assume that $a_{2}(G)<0$. Since $s(I)=2$, by Theorem 2.1 we get that $r\left(I^{n}\right)=1$ for $n \gg 0$. For such a number $n R\left[I^{n} t\right]$ is C-M (by [10, Proposition 7.2]).

Example 3.6. Let

$$
R=K\left[\left[u^{4}, u^{3} v, u^{2} v^{2}, u v^{3}, v^{4}\right]\right] \text { and } I=\left(u^{4}, u^{3} v, u v^{3}, v^{4}\right) R .
$$

Then $R$ is a C-M ring, $H_{G_{+}}^{0}(G) \cong K, H_{G_{+}}^{1}(G) \cong K(-1)$, and $\left[H_{G_{+}}^{2}(G)\right]_{n}=0$ for $n \geq 0$. By [10, Theorem 1.1] it then follows that $R[I t]$ is not C-M, but $R\left[I^{n} t\right]$ is C-M for all $n \geq 2$. This example also shows that the converse of (i) of Theorem 3.1 is not true.

We denote by $\operatorname{edim} R$ and $e(R)$ the embedding dimension and the multiplicity of $R$, resp. The following result extends [4, Proposition 1.4].

Corollary 3.7. Let $(R, \mathfrak{m})$ be a 2-dimensional ring. Then $R\left[\mathfrak{m}^{n} t\right]$ is $C-M$ for some $n>0$ (or for all $n>0$ ) if and only if $R$ is a $C-M$ ring with $\operatorname{edim} R=$ $e(R)+1$.

Proof. Proposition 1.4 of [4] states that $R[\mathrm{~m} t]$ is C-M (hence so is $R\left[\mathrm{~m}^{n} t\right]$ for all $n>0$ ) if and only if $R$ is a C-M ring with edim $R=e(R)+1$. Therefore, it suffices to show that the last condition also follows from Cohen-Macaulayness of $R\left[\mathfrak{m}^{n} t\right]$ for some $n>0$. Indeed, by Proposition 3.4, $R$ is C-M. By Corollary 3.5 and Theorem 2.1, $r\left(\mathrm{~m}^{k}\right)=1$ for $k \gg 0$. Hence it follows from [13, Corollary 4.4] that $\operatorname{edim} R=e(R)+1$.

\section{REFERENCES}

1. M. Brodmann, Local cohomology of certain Rees- and form-rings. II, J. Algebra 86 (1984), 457-493.

2. D. Eisenbud and S. Goto, Linear free resolutions and minimal multiplicity, J. Algebra 88 (1984), 89-133.

3. M. Fiorentini and L. T. Hoa, Some remarks on generalized Cohen-Macaulay rings, Bull. Soc. Math. Belg. (to appear).

4. S. Goto and Y. Shimoda, On the Rees algebras of Cohen-Macaulay rings, Commutative Algebra (Fairfax 1979), Lecture Notes in Pure and Appl. Math., vol. 68, Dekker, New York, 1982.

5. S. Goto and K. Watanabe, Graded rings. I, J. Math. Soc. Japan 30 (1978), 179-213.

6. M. Herrmann, J. Ribbe, and S. Zarzuela, On the Gorenstein property of Rees and form rings of powers of ideals, Trans. Amer. Math. Soc. (to appear).

7. J. Herzog, A. Simis, and W. V. Vasconcelos, On the canonical modules of the Rees algebra and the associated graded ring of an ideal, J. Algebra 105 (1987), 285-302.

8. S. Huckaba, Reduction numbers for ideals of higher analytic spread, Math. Proc. Cambridge Philos. Soc. 102 (1987), 49-57.

9. __ On complete $d$-sequences and the defining ideals of Rees algebras, Math. Proc. Cambridge Philos. Soc. 106 (1989), 445-458. 
10. S. Ikeda and N. V. Trung, When is the Rees algebra Cohen-Macaulay? Comm. Algebra 17 (1989), 2893-2922.

11. D. G. Northcott and D. Rees, Reductions of ideals in local rings, Math. Proc. Cambridge Philos. Soc. 50 (1954), 145-158.

12. A. Ooishi, Castelnuovo's regularity of graded rings and modules, Hiroshima Math. J. 12 (1982), 627-644.

13. __ Stable ideals in Gorenstein local rings, J. Pure Appl. Algebra 69 (1990), 185-191.

14. J. D. Sally, Tangent cones at Gorenstein singularities, Compositio Math. 40 (1980), 167175.

15. __ Reductions, local cohomology and Hilbert functions of local rings, Commutative Algebra (Durham 1981), London Math. Soc. Lecture Note Ser., vol. 72, Cambridge Univ. Press, Cambridge and New York, 1982.

16. J. D. Sally and W. V. Vasconcelos, Stable rings, J. Pure Appl. Algebra 4 (1974), 319-336.

17. P. Schenzel, Castelnuovo's index of regularity and reduction numbers, Topics in Algebra, part 2, Banach Center Publication, vol. 26, PWN, Warsaw, 1990, pp. 201-208.

18. N. V. Trung, Towards a theory of generalized Cohen-Macaulay modules, Nagoya Math. J. 102 (1986), 1-49.

19. _ Reduction exponent and degree bound for the defining equations of graded rings, Proc. Amer. Math. Soc. 101 (1987), 229-236.

Institute of Mathematics, P.O. Box 631, Boho Hanoi, Vietnam

Current address: Centre de Recerca Matematica, Institut d'Estudis Catalans, 08193 BellaterraBarcelona, Spain

E-mail address: ICRME@EBCCUAB1 